\title{
Angiotensin-converting enzyme inhibitors and angiotensin receptor blockers in high vascular risk
}

\author{
Louis Potier, ${ }^{1,2,3}$ Ronan Roussel, ${ }^{1,2,3}$ Yedid Elbez, ${ }^{4}$ Michel Marre, $1,2,3$ Uwe Zeymer, ${ }^{5}$ \\ Christopher M Reid, ${ }^{6}$ Magnus Ohman, ${ }^{7}$ Kim A Eagle, ${ }^{8}$ Deepak L Bhatt, ${ }^{9}$ \\ Philippe Gabriel Steg, ${ }^{2,4,10}$ on behalf of the REACH Registry Investigators*
}

\begin{abstract}
- Additional material is
published online only. To view please visit the journal online (http://dx.doi.org/10.1136/ heartjnl-2016-310705).
\end{abstract}

For numbered affiliations see end of article.

Correspondence to Dr Louis Potier, Department of Diabetology, Endocrinology and Nutrition, Bichat-Claude Bernard Hospital, 46 rue Henri Huchard, Paris 75018, France; louis.potier@gmail.com

Received 29 September 2016 Revised 30 January 2017 Accepted 3 February 2017

\section{Linked}

- http://dx.doi.org/10.1136/ heartjinl-2017-311331

\section{CrossMark}

To cite: Potier L, Roussel R, Elbez Y, et al. Heart 2017;103:1339-1346.

\section{ABSTRACT}

Objective ACE inhibitors (ACEls) and angiotensin receptor blockers (ARBs) are widely prescribed in patients with high cardiovascular (CV) risk. However, whether both classes have equivalent effectiveness to prevent $\mathrm{CV}$ events remains unclear. The aim of this study was to compare the incidence of major CV events between ACEI and ARB users.

Methods The Reduction of Atherothrombosis for Continued Health registry is an observational study who enrolled 69055 individuals with high CV risk. Among them, 40625 patients (ACEls $67.9 \%$ and ARBs 32.1\%) were included. Main outcome was rates of CV mortality, non-fatal myocardial infarction, non-fatal stroke or hospitalisation for CV disease at 4 years.

Results In a propensity score-adjusted cohort, the incidence of the primary outcome was lower in patients on ARBs compared with ACEIs (29.2\% vs $33.4 \%$; adjusted HR 0.90; $95 \% \mathrm{Cl} 0.86$ to $0.95 ; \mathrm{p}<0.001)$. Similar results were observed for CV $(6.9 \%$ vs $8.2 \%$; HR 0.83; $95 \% \mathrm{Cl} 0.75$ to $0.93 ; p=0.001)$ and allcause mortality $(11.6 \%$ vs $12.6 \%$; HR $0.89 ; 95 \% \mathrm{Cl}$ 0.82 to $0.97 ; p=0.005)$. Analyses using propensity score matching yielded similar results. History of diabetes or estimated glomerular filtration rate did not affect the results. ARB use was associated with lower rates of all-cause mortality in secondary prevention but not in primary prevention patients ( $p$-value for interaction=0.03).

Conclusion ARB use appears to be associated with $10 \%$ lower rates of CV events compared with ACEls, especially in patients with established CV disease. Our results suggest that ARBs may provide superior protection against CV events than ACEls in high-risk patients in real-world practice.

\section{INTRODUCTION}

ACE inhibitors (ACEIs) and angiotensin receptor blockers (ARBs) are widely prescribed in patients with a high cardiovascular (CV) risk. Both classes target the renin-angiotensin system (RAS) but have different sites of action. ${ }^{1}$ Potential difference in comparative efficacy of ACEIs and ARBs is a subject of debate. ${ }^{2}$ Both American and European guidelines for the management of patient with CV disease recommend ACEIs as a first choice therapy, whereas ARBs are an alternative therapy for ACEI-intolerant patients. ${ }^{34}$ These recommendations are based on the marked benefit of ACEIs in randomised clinical trials (RCTs) compared with no consistent benefit of ARBs versus placebo in stable $\mathrm{CV}$ disease. However, a recent meta-analysis highlighted ARBs to be as efficacious and safe as ACEIs when analysis was restricted to trials after the year 2000, when aggressive prevention therapies were routinely used. ${ }^{2}$ Moreover, no difference between the two classes was observed in recent head-to-head RCTs of ACEIs versus ARBs, ${ }^{5-9}$ but these studies were limited to highly selected patients, and their extrapolation to the average patient may be debatable. ${ }^{10}$ Conversely, observational studies could provide a better external validity than RCTs to evaluate the effect of long-term use of drugs in realworld populations. ${ }^{11} 12$ For example, analyses from the Reduction of Atherothrombosis for Continued Health (REACH) cohort challenged the common views on efficacy of $\beta$-blockers or renin-angiotensin antagonists in subjects with stable coronary artery disease. ${ }^{13}{ }^{14}$ Finally, recent meta-analyses that assessed efficacy of blood pressure-lowering agents on $\mathrm{CV}$ outcomes in patients with hypertension or diabetes reported conflicting results regarding comparisons between ACEIs and ARBs. ${ }^{15-18}$

The REACH registry provides an opportunity to directly compare ACEIs' and ARBs' effectiveness in routine clinical practice, in a contemporary large cohort of outpatients with high CV risk, with a variety of comorbidities, broad geographic representation and under well-defined intensity of preventive therapy. Data from this cohort were used to compare the incidence of mortality and CV events according to the use of ACEIs or ARBs.

\section{METHODS}

\section{Study population}

The design, methods and primary results of the international REACH registry have been published previously. ${ }^{19}$ Briefly, 69055 patients at least 45 years old with $\geq 3$ risk factors for atherosclerosis and patients with previous CV disease were enrolled between 2003 and 2004. Risk factors taken into account included history of diabetes, diabetic nephropathy, ankle-brachial index $\leq 0.9$, asymptomatic carotid stenosis of $\geq 70 \%$, carotid intima media thickness at least two times that at adjacent sites, systolic blood pressure $\geq 150 \mathrm{~mm} \mathrm{Hg}$ despite treatment, hypercholesterolaemia treated with medication, current smoking of $\geq 15$ cigarettes per day and age $\geq 65$ years for men or $\geq 70$ years for women. Signed informed consent was obtained from all patients, and the institutional review board 


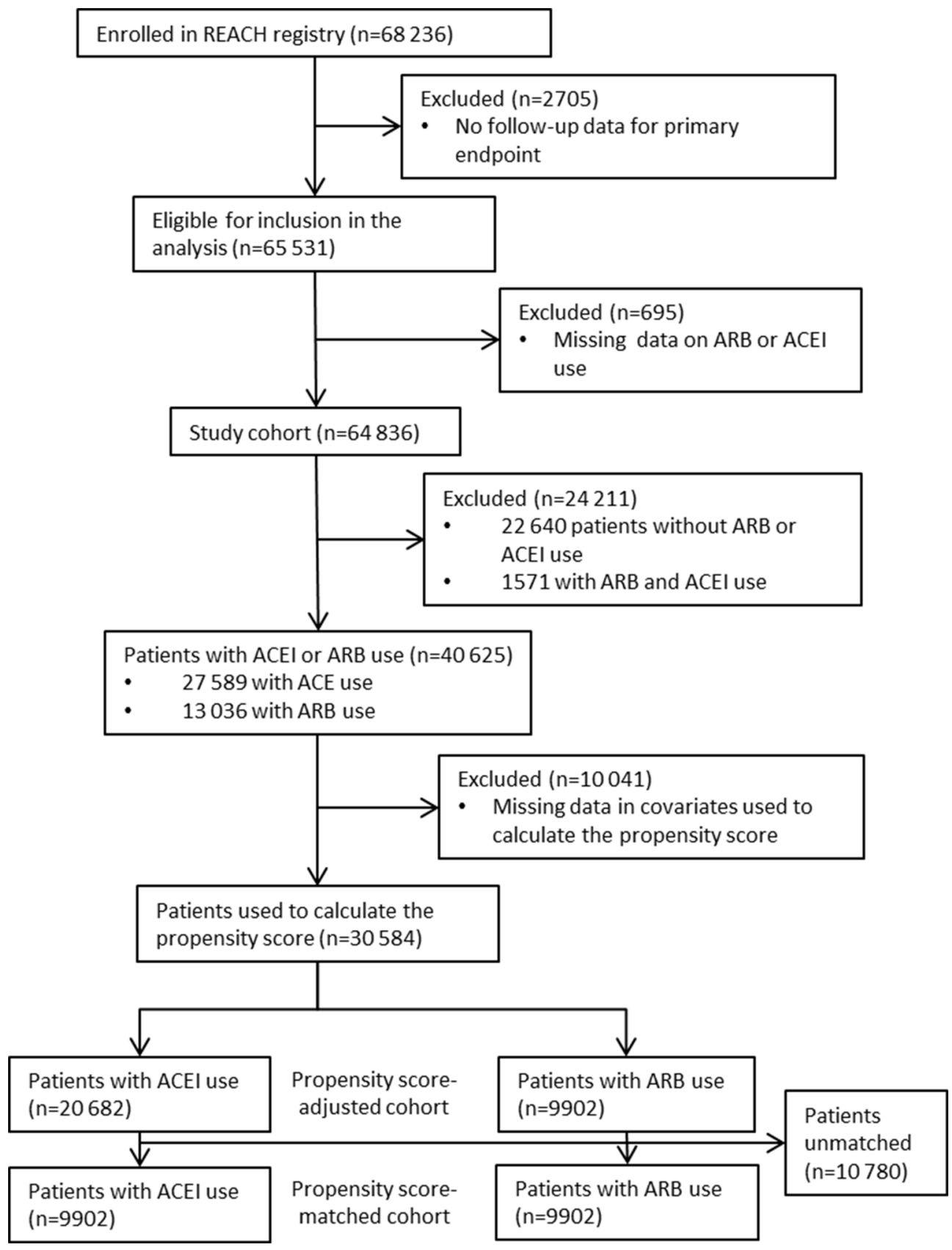

Figure 1 Flow diagram. ACEl, ACE inhibitor; ARB, angiotensin receptor blocker; REACH, Reduction of Atherothrombosis for Continued Health.

in each country approved the protocol according to the local requirements. These patients were assessed annually at years 1 through 4, and follow-up was completed in 2008. Patients included in the present analysis were those with complete 4-year follow-up information and ACEI or ARB status at baseline. Patients taking a combination of ACEI and ARB were excluded from the analysis (figure 1).

\section{Outcomes measures}

The primary outcome was the composite of CV mortality, non-fatal myocardial infarction (MI), non-fatal stroke or hospitalisation for $\mathrm{CV}$ reasons during the 4-year follow-up. Individual components of the primary outcome, all-cause mortality and heart failure (HF) (defined by signs or symptoms of left or right ventricular failure or both) were also studied.

\section{Statistical analysis}

Continuous variables are expressed as mean (SD), and categorical variables are expressed as frequencies and percentages. To account for differences in baseline characteristics between treatment groups (see online supplementary table 1), propensity score analysis was performed to further account for these differences between ACEIs and ARBs users.

To calculate propensity score, 10041 patients were excluded due to missing data in covariates used to calculate the propensity score. No major difference was observed between patients included and excluded to calculate propensity score (see online supplementary table 2). Propensity scores for all patients were first estimated using a non-parsimonious multivariable logistic regression model, with ACEIs or ARBs use as the dependent variable and 17 baseline characteristics as covariates (sex, 
age, estimated glomerular filtration rate (eGFR), geographical regions (divided in two groups), current smoker status, hypertension, history of HF, history of atrial fibrillation, history of coronary artery disease, history of MI, history of diabetes, aspirin use, statin use, beta blockers use, calcium channel blockers use, nitrates use and diuretics use).

Cox models for primary and secondary outcomes were conducted with ACEI versus ARB status as covariate after adjustment on propensity score and an additional analysis was performed by matching ARBs users with ACEIs users, based on their propensity score. The matching was performed by a SAS macro ${ }^{20}$ implementing an algorithm based on 8 to 1 digit matching (ratio of 1:1 without replacement). For ACEI user, ARB user with similar propensity scores was selected. This matched couple was thus removed from the cohort, and the matching procedure was iteratively rerun until a maximum number of patient couples were assembled. We were able to match 19804 patients, of which 9902 patients on ARBs (76\% of the ARB cohort) and 9902 patients on ACEIs (35.9\% of the ACEI cohort).

Absolute standardised differences for all covariates before and after matching were estimated to evaluate bias reduction using the propensity score matching method. After propensity score matching, all absolute standardised differences were $<10 \%$, indicating robust matching (see online supplementary figure 1). Statistical tests of comparisons in baseline characteristics between the matched groups were also non-significant $(\mathrm{p}>0.05)$ (see online supplementary table 3 ).

In the propensity score-adjusted cohort, Cox proportional hazard regression model with the propensity score introduced as a covariate was used to compare the risk of outcomes between both groups. In the propensity score-matched cohort, we used cox proportional hazard regression model stratified on the matched pairs.

Subgroup analyses were also performed in the propensity score-adjusted and score-matched cohorts according to diabetes, eGFR values (divided into three groups: $\leq 30,>30$ and $\leq 60$, $>60 \mathrm{~mL} / \mathrm{min}$ ), established arterial disease and $\mathrm{HF}$ at baseline. We also compared ACEIs and ARBs according to the numbers of medication use in addition to ACEIs or ARBs and believed to reduce the incidence of CV events: optimal medical therapy, that is, statins, $\beta$-blockers and antiplatelet agents, including aspirin.
Heterogeneity across subgroups was tested by introducing interaction terms into the Cox proportional hazard regression models.

Proportional hazard assumptions were checked by testing the interaction between ACEI versus ARB status and log (time) within the model.

Analysis was performed using SAS version 9.3 (SAS Institute, Cary, North Carolina).

\section{RESULTS}

\section{Study population}

From the 68236 patients with available baseline data, 65531 were enrolled for 4-year follow-up. Among them, 40625 patients (70.0\%) had ACEI or ARB user status. Patients were divided into two groups according to ACEI or ARB use at baseline (27589 (67.9\%) ACEI-only users, 13036 (32.1\%) ARBonly users) (figure 1).

Among those patients who used ACEI or ARB at baseline, discontinuation was similar: the proportion of patients remaining on ACEIs or ARBs was $88.3 \%$ and $87.7 \%$ at 12 months, $84.9 \%$ and $84.5 \%$ at 2 years, $80.0 \%$ and $81.5 \%$ at 3 years, and $76.8 \%$ and $78.8 \%$ at 4 years, respectively.

\section{Study outcomes}

In the propensity score-adjusted cohort, at least one of the components of the primary outcome occurred in 7788 patients during the 4-year follow up (cumulative incidence rate of 32.01\%). Compared with ACEI users, the rate of the primary outcome was lower in ARB users (adjusted HR 0.90; 95\% CI 0.86 to $0.95 ; \mathrm{p}<0.001$ ) (figure 2 ). The individual components of the primary endpoint and all-cause mortality were lower in ARBs users for CV mortality (HR 0.83; 95\% CI 0.75 to 0.93 ; $\mathrm{p}=0.001$ ), hospitalisation for $\mathrm{CV}$ reasons (HR $0.91 ; 95 \%$ CI 0.85 to $0.96 ; \mathrm{p}<0.001)$ and all-cause mortality $(\mathrm{HR} 0.89 ; 95 \% \mathrm{CI}$ 0.82 to $0.97 ; \mathrm{p}=0.005$ ). There was no difference in the other components of the primary outcome (non-fatal MI, non-fatal stroke) in the propensity score-adjusted cohort. HF occurred in 2473 patients during the 4-year follow up and was similar between the two groups $(8.49 \%$ and $7.24 \%$ in ACEI and ARB groups, respectively; HR $0.96,95 \% \mathrm{CI} 0.88$ to 1.06 ; $\mathrm{p}=0.418$ ).

Similar results to the propensity score-matched cohort were observed. The adjusted HR was 0.91 (95\% CI 0.85 to 0.97 ;

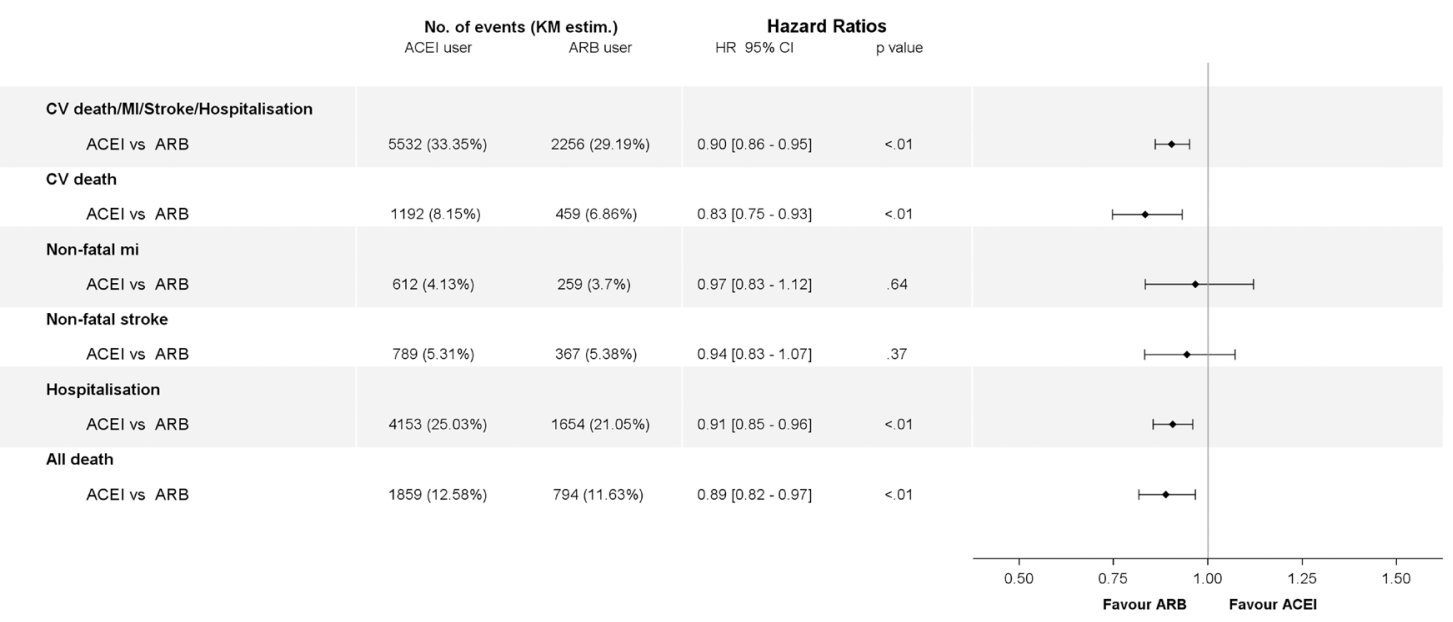

Figure 2 Risks of outcomes in ACEls versus ARBs cohorts, in propensity score-adjusted analysis. ACEls, ACE inhibitors; ARB, angiotensin receptor blockers; CV, cardiovascular; MI, myocardial infarction. 


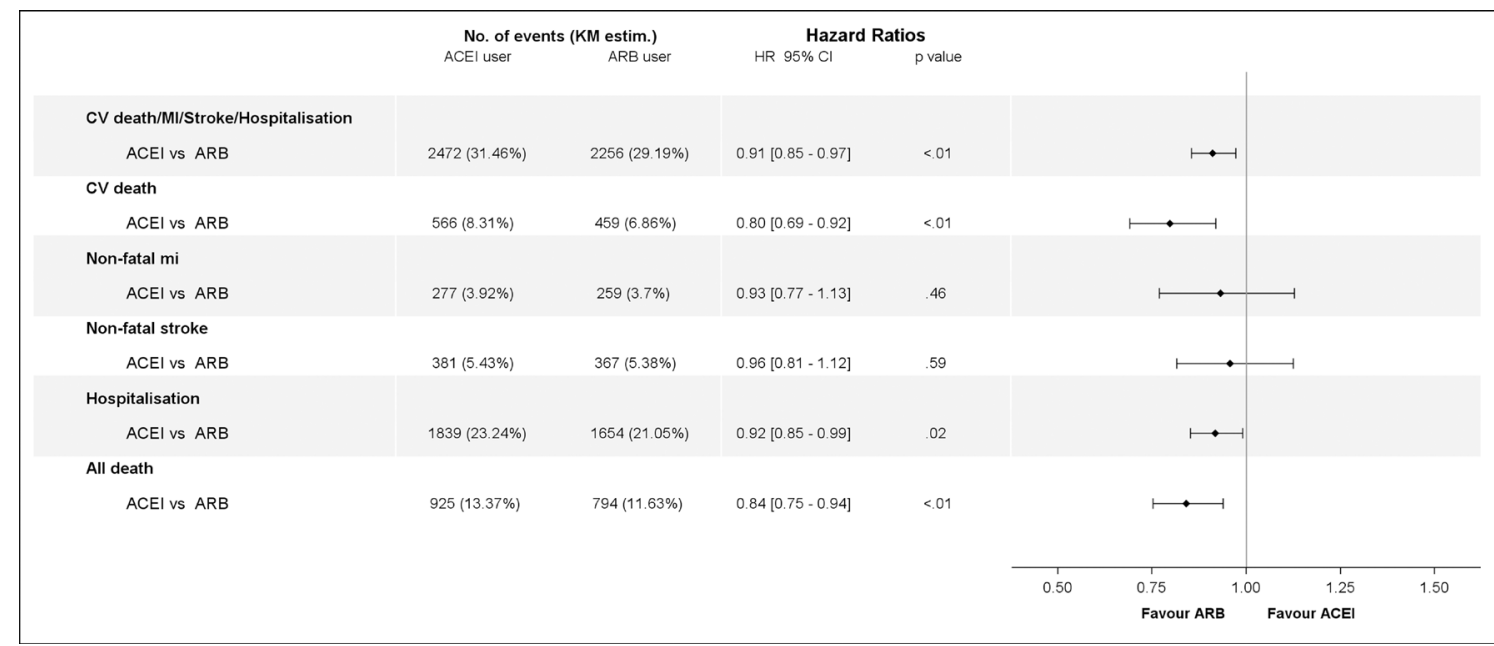

Figure 3 Risks of outcomes in ACEls versus ARBs cohorts, in propensity score-matched analysis. ACEls, ACE inhibitors; ARB, angiotensin receptor blocker; CV, cardiovascular; MI, myocardial infarction.

$\mathrm{p}=0.005)$ for the primary outcome, 0.80 (95\% CI 0.69 to 0.92 ; $\mathrm{p}=0.001)$ for C mortality and 0.84 (95\% CI 0.75 to 0.94 ; $\mathrm{p}=0.002$ ) for all-cause mortality (figure 3 ).

\section{Subgroup analysis}

In both cohorts, history of diabetes at baseline or level of eGFR did not affect the primary results. Lower rates of primary outcome, CV mortality, hospitalisation for CV reasons or all-cause mortality were observed in ARB users compared with
ACEI users (figures 4-7). However, in patients without established atherosclerosis (primary prevention), there was no difference between ARB or ACEI users for all outcomes, whereas in established atherosclerosis (secondary prevention) ARB use was associated with a reduction of the primary outcome, CV mortality, hospitalisation for CV reasons or all-cause mortality, although the interaction was significant only for CV mortality in the propensity score-matched and adjusted cohort ( $p$ value for interaction $=0.03$ in both cohorts).

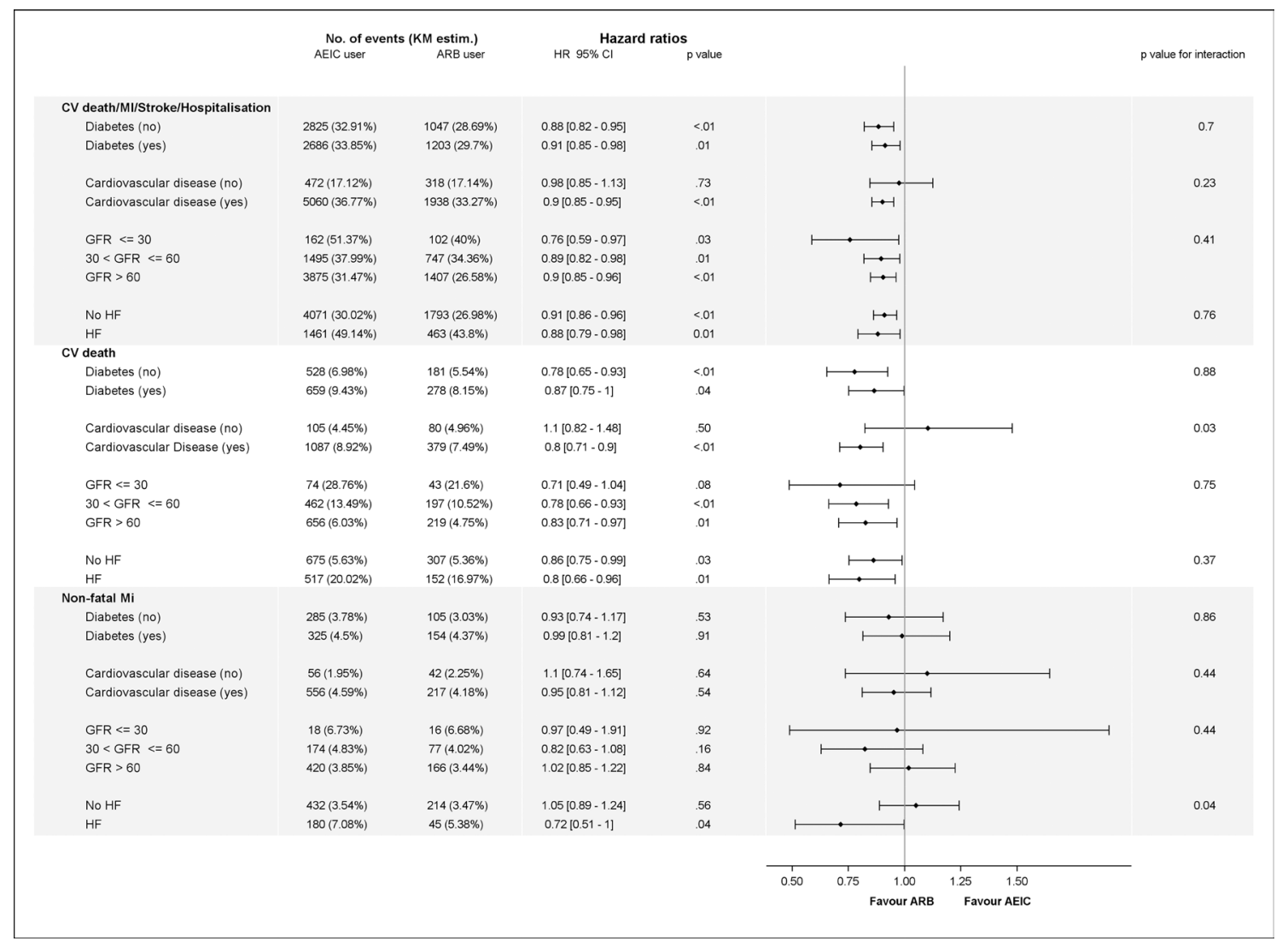

Figure 4 Risks of outcomes (primary outcomes, CV death, Non-fatal MI) in ACEls versus ARBs cohorts in subgroups, in propensity score-adjusted analysis. ACEls, ACE inhibitors; ARB, angiotensin receptor blocker; CV, cardiovascular; MI, myocardial infarction; HF, heart failure; GFR, glomerular filtration rate. 


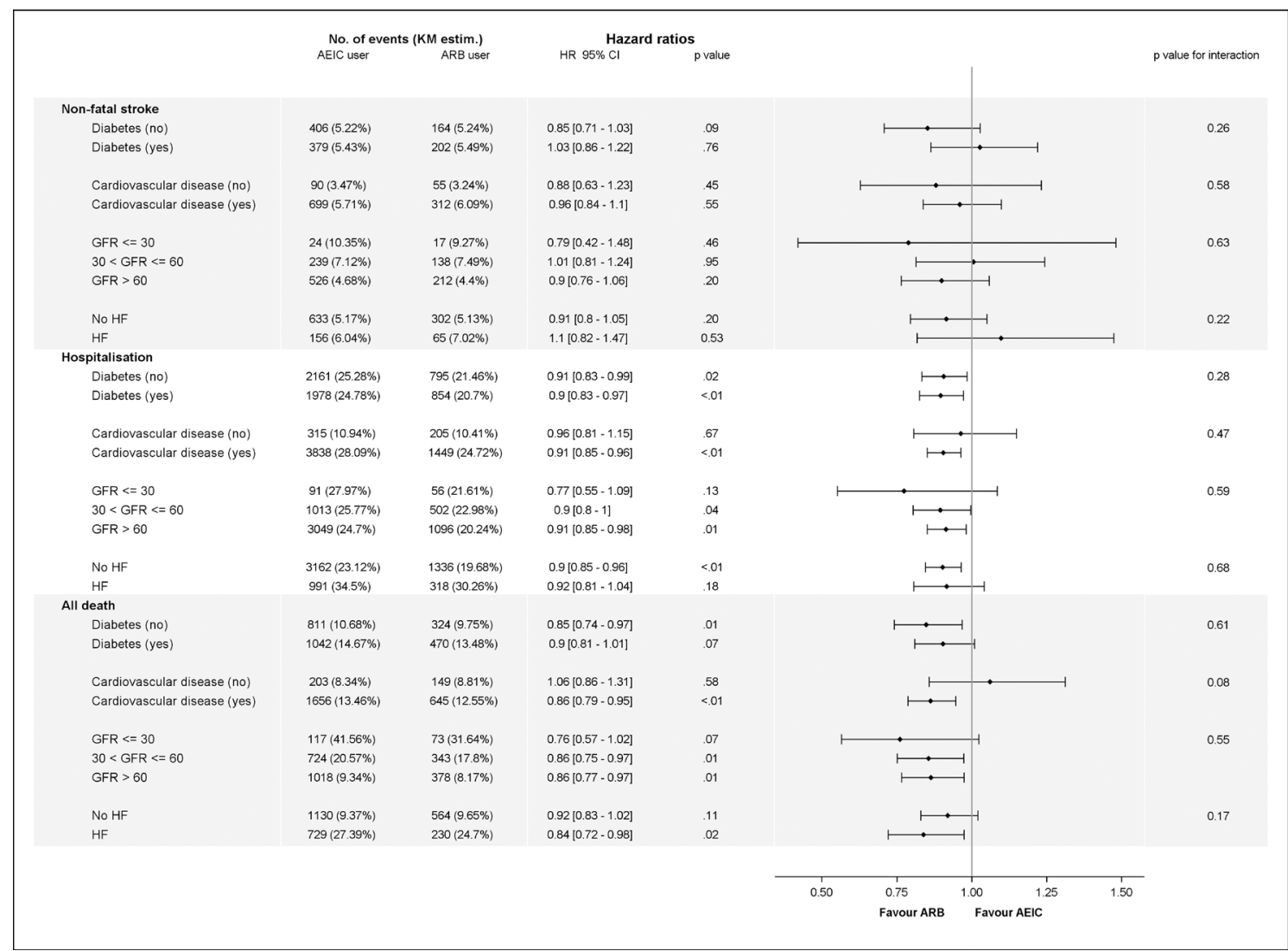

Figure 5 Risks of outcomes (non-fatal stroke, hospitalisation, all death) in ACEls versus ARBs cohorts in subgroups, in propensity score-adjusted analysis. ACEIs, ACE inhibitors; ARB, angiotensin receptor blocker; CV, cardiovascular; MI, myocardial infarction; HF, heart failure; GFR, glomerular filtration rate.

Similarly, in both cohorts, ARB use was associated with a lower rate of non-fatal MI in patients with history of HF but not in patients without HF (figures 4-7). This effect of ARBs or ACEIs for non-fatal MI interacted significantly in both cohorts ( $\mathrm{p}$ value for interaction $=0.04$ and 0.01 in propensity score-adjusted and matched cohorts, respectively). Otherwise, history of $\mathrm{HF}$ at baseline did not affect the similar rate of HF during follow-up in patients taking ACEIs and ARBs (data not shown).

We compared ACEIs and ARBs according to the use of optimal medical therapy for the prevention of CV disease. As expected, CV or all-cause mortality decreased with the number of treatments used (see online supplementary figures 2, 3) in both cohorts. In the propensity score-adjusted cohort, the difference between ACEIs and ARBs for primary outcomes, CV and all-cause mortality was gradually attenuated with the optimisation of CV treatments. This attenuation was significant for CV and all-cause mortality ( $p$ value for interaction $<0.01$ in both parameters).

\section{DISCUSSION}

In this analysis of 40625 people from the REACH registry, we found that, in comparison with ACEI use, routine use of ARBs was associated with a lower rate of major CV events in outpatients with high CV risk. We observed a consistency of our findings after multiple adjustment methods. Moreover, subgroup analysis found similar results regardless of a history of diabetes, HF or chronic kidney disease. However, the difference between $\mathrm{ARB}$ and ACEI use was only significant in the subgroup of patients with established atherosclerosis, with a significant interaction for all-cause mortality.
In most RCTs, ACEIs and ARBs have been associated with decreased major CV events compared with placebo or active comparators, including in patients with high $\mathrm{CV}$ risk. $^{21-24}$ However, due to the different mechanisms of action of these two classes of drugs, differential clinical effect could be expected. ${ }^{1}$ Indeed, in the meta-analysis of Van Vark et al, treatment with ACEIs, but not ARBs, reduced mortality in patients with hypertension. ${ }^{15}$ Likewise, a recent network meta-analysis concluded that ACEIs reduced all-cause mortality, CV mortality and major $\mathrm{CV}$ events, whereas ARBs had no benefits on these outcomes in patients with diabetes. ${ }^{16}$ However, a recent meta-analysis pointed out several differences in the ACEIs versus placebo and ARBs versus placebo trials. ${ }^{2}$ One was that most of trials with ACEIs were conducted a decade earlier than the ARB trials with a lower placebo event rate, which reflected the concomitant optimised medication use in the ARB trials versus the ACEI trials. In that report, when analyses were restricted to trials published after the year 2000, outcomes were similar between ACEIs versus placebo and ARBs versus placebo. In line with these results, when we analysed the interaction between ACEI/ $\mathrm{ARB}$ and quality of therapy, we found a significant attenuation of the beneficial effect of ARBs versus ACEIs on mortality with the optimisation of medical therapy.

In the few head-to-head RCTs that compared ACEIs and ARBs, no difference was found between both classes. However, it is noteworthy that in the three oldest studies, doses of ARB used were lower than the higher dose recommended. ${ }^{525} 26$ Moreover, the neutral results of the three recent head-to-head trials may be a consequence of a more aggressive use of a background optimal medical therapy, as concomitantly recommended. ${ }^{6-8}$ Another 


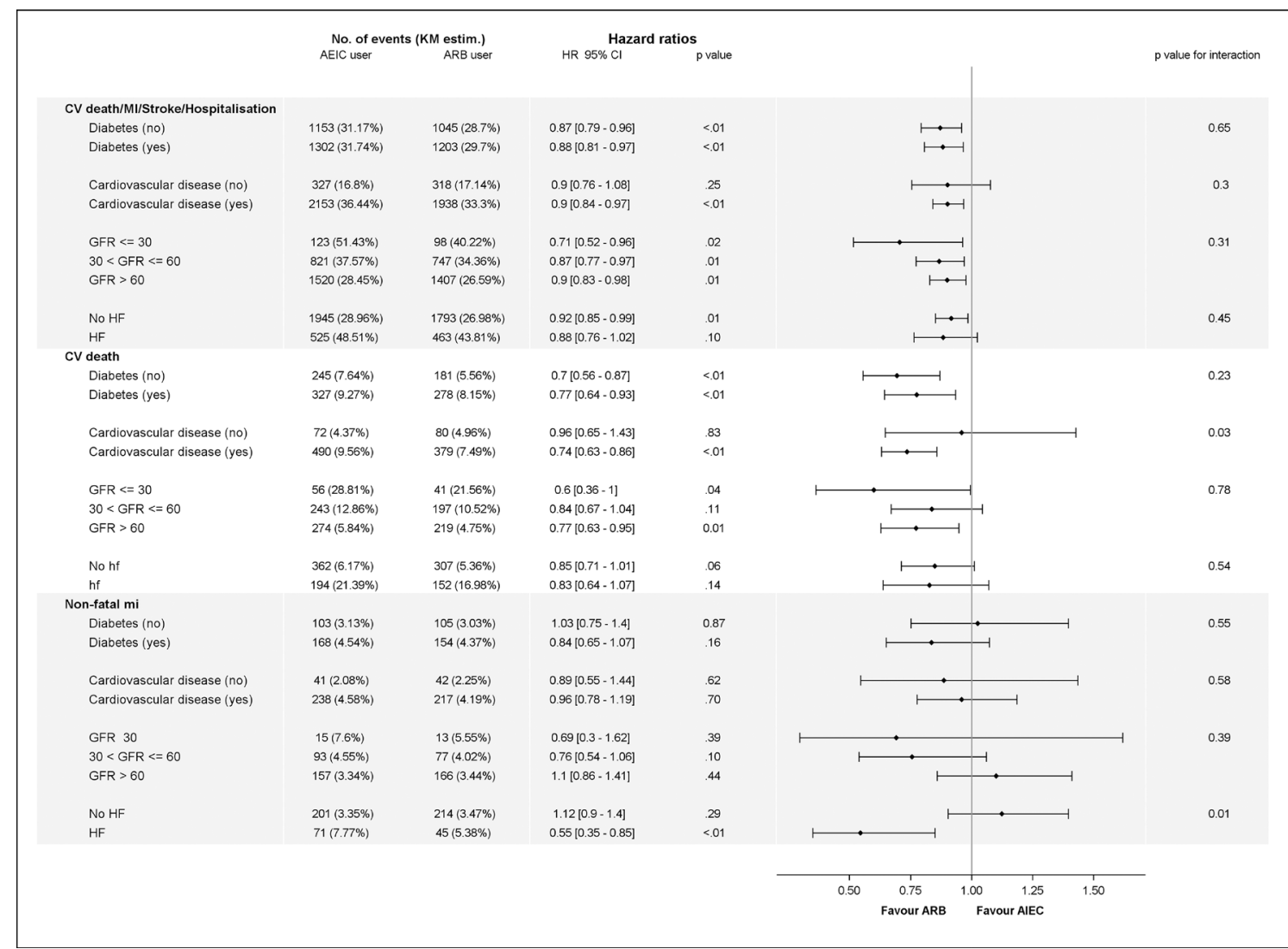

Figure 6 Risks of outcomes (primary outcomes, CV death, non-fatal MI) in ACEls versus ARBs cohorts in several subgroups, in propensity scorematched analysis. ACEls, ACE inhibitors; ARB, angiotensin receptor blocker; CV, cardiovascular; MI, myocardial infarction; HF, heart failure; GFR, glomerular filtration rate.

difference in ACEIs and ARBs trials was that more patients with a history of coronary or other vascular disease were included in ACEI trials than in ARB trials. ${ }^{2}$ These differences could also explain discrepancies between these previous meta-analyses and our results, suggesting that ARBs could be more beneficial than ACEIs in patients with a prior history of $\mathrm{CV}$ disease. Furthermore, a recent meta-analysis of the association between blood pressure-lowering treatments and vascular disease in patients with type 2 diabetes showed that ARBs were associated with a significant reduction in mortality compared with any active comparator including ACEIs. ${ }^{17} \mathrm{~A}$ similar trend was observed with ARBs, but not with ACEIs, on all-cause mortality in patients with diabetes and kidney disease in the network meta-analysis of Palmer et al. ${ }^{18}$ It is noteworthy that in most of these meta-analyses, moderate or higher heterogeneity between populations included in trials limited the validity of the pooled analysis. Moreover, results from RCTs comparing ACEIs or ARBs with active controls showed that the blood pressure-lowering independent effect of both classes are less evident in patients without previous CV disease than in secondary $\mathrm{CV}$ prevention population. ${ }^{2}$ This observation could explain the lack of difference between ACEI and ARB use observed in our primary prevention population.

In contrast to our results on primary outcomes and all-cause mortality, lack of difference on incident HF between the two groups in the whole population and in subgroups of patients with or without HF at baseline was surprising. Moreover, as mentioned in the 2016 ESC guidelines on the management of patients with HF, ARBs are recommended only as an alternative in patients intolerant of an ACEI. ${ }^{27}$ This recommendation is based on the fact that there are more RCTs supporting the use of ACEIs than of
ARBs. However, our findings were in line with previous head-tohead trials in patients with or without HF at baseline in which no difference on HF between both classes was observed. ${ }^{6-8} 26$

It would have been interesting to study the effect of a combination of ARBs and ACEIs at baseline. Unfortunately, the small size of this subgroup (1571 patients) did not allow to perform any convincing propensity score-based analysis.

Despite our findings are derived from a large observational cohort with a 4-year follow-up in a broad range populations, our analysis has limitations. Although propensity score adjustment, the potential for residual confounding by indication due to unmeasured or measured confounders, cannot be completely ruled out. However, reproducibility of the results across several models, subgroups and adjustment methods used suggest that these results are valid. Moreover, the size of the population and the attendant narrow CIs of clinical outcome rates reinforce the strength of our findings. Another important limitation was we did not have data regarding doses of ACEIs and ARBs used. It is well known that the beneficial effect of RAS antagonists observed in RCTs is obtained from using higher doses of ACEIs or ARBs ${ }^{2128-30}$ whereas doses used in routine clinical practice are often lower. Likewise, this study cannot take into account the differences that may exist between drugs within the same class due to the lack of this information.

In conclusion, in the large international REACH registry, ARB use appears associated with lower rates of major CV outcomes compared with ACEI use, especially in patients with established CV disease. Despite a greater level of evidence for ACEIs than for ARBs from RCTs and a similar effect of both classes in head-tohead RCTs, these results question the differential effect of ACEIs 


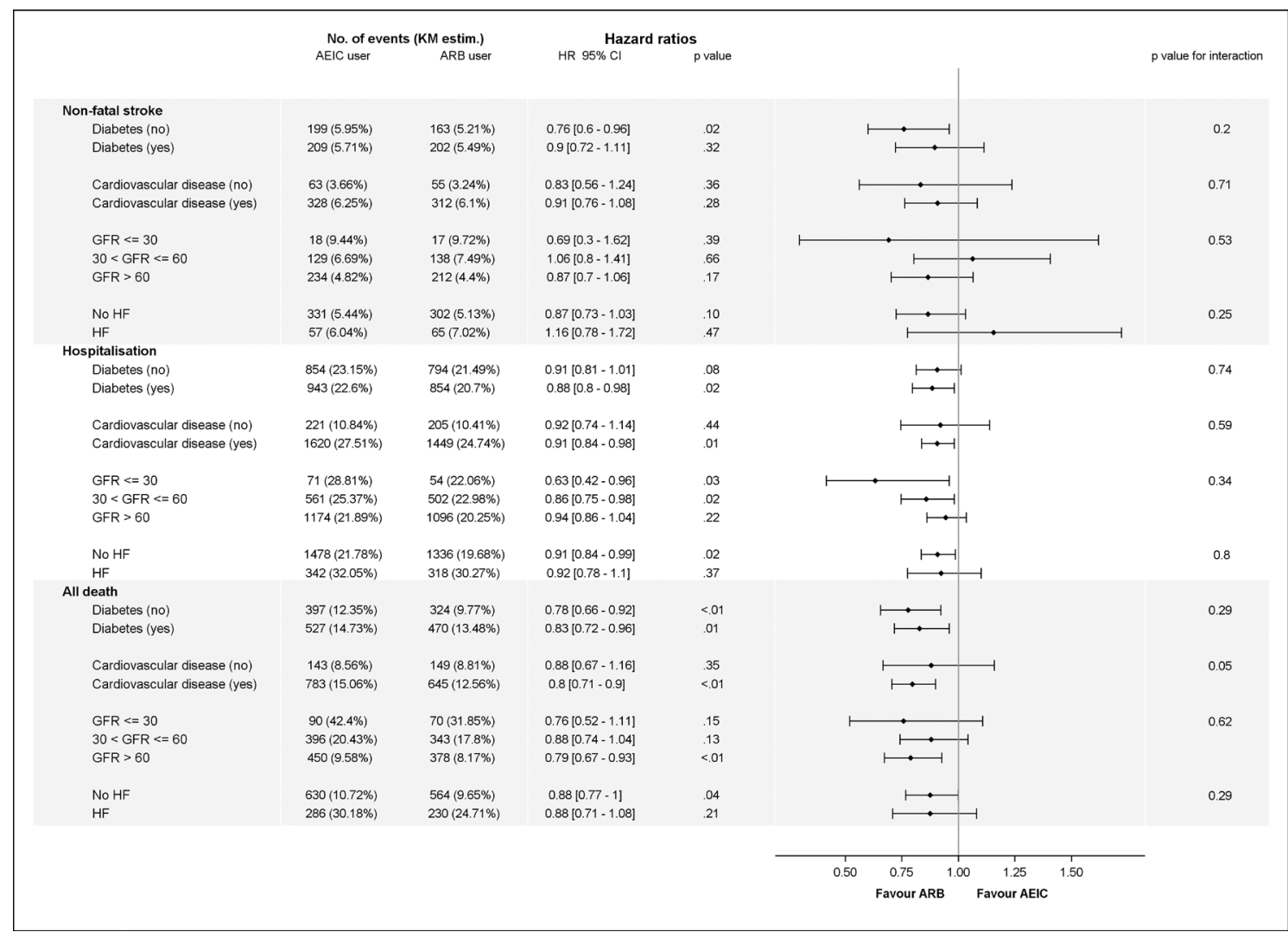

Figure 7 Risks of outcomes (non-fatal stroke, hospitalisation, all death) in ACEls versus ARBs cohorts in several subgroups, in propensity scorematched analysis. ACEIs, ACE inhibitors; ARB, angiotensin receptor blocker; CV, cardiovascular; MI, myocardial infarction; HF, heart failure; GFR, glomerular filtration rate.

and ARBs in patients in secondary prevention in actual clinical practice.

\section{Key messages}

\section{What is already known on this subject?}

Both American and European guidelines for the management of patient with cardiovascular disease recommend ACE inhibitors (ACEIs) as a first-choice therapy whereas angiotensin receptor blockers (ARBs) are an alternative therapy for ACEl-intolerant patients. However, data available from RCTs and meta-analyses reported conflicting results regarding comparisons between ACEls and ARBs. Whether ACEls and ARBs have equivalent effectiveness to prevent cardiovascular events remains unclear.

\section{What might this study add?}

Our results from a contemporary large cohort of outpatients with high cardiovascular risk show that the incidence of major adverse cardiovascular events was significantly lower in patients on ARBs compared with ACEls (29.2\% vs $33.4 \%$; adjusted HR $0.90 ; 95 \% \mathrm{Cl} 0.86$ to $0.95 ; \mathrm{p}<0.001)$, especially in patients with established cardiovascular disease.

\section{How might this impact on clinical practice?}

Our results suggest that ARBs should be preferred to ACEls in patients with a previous history of cardiovascular diseases.

\section{Author affiliations}

'Département d'Endocrinologie, Diabétologie et Nutrition, DHU-FIRE, HUPNVS, APHP, Paris, France

¿Université Paris Diderot-Sorbonne Paris Cité, Paris, France
${ }^{3}$ Centre de Recherche des Cordeliers, INSERM, U-1138, Paris, France ${ }^{4}$ FACT DHU FIRE, LVTS INSERM U-1148, Hôpital Bichat, HUPNVS, AP-HP, Paris, France ${ }^{5}$ Klinikum Ludwigshafen und Institut für Herzinfarktforschung, Ludwigshafen, Rhineland-Palatinate, Germany

${ }^{6}$ Department of Epidemiology and Preventive Medicine, Monash University, Alfred Hospital, School of Public Health, Curtin University, Centre of Cardiovascular Research and Education in Therapeutics, Melbourne, Perth, Australia

${ }^{7}$ Division of Cardiology, Department of Medicine, Duke University Medical Center and the Duke Clinical Research Institute, Durham, North Carolina, USA

${ }^{8}$ Department of Cardiology, University of Michigan Health System, Ann Arbor, Michigan, USA

${ }^{9}$ Brigham and Women's Hospital Heart \& Vascular Center and Harvard Medical School, Boston, Massachusetts, USA

${ }^{10} \mathrm{NHLI}$ Imperial College, Royal Brompton Hospital, London, UK

Contributors Drafting the work: $L P, R R, Y E$; Revising the work critically for important intellectual content: YE, MM, UZ, CMR, EMO, KAE, DLB, PGS; Substantial contributions to the conception or design of the work, and the acquisition of data: UZ, CMR, EMO, KAE, DLB and PGS; Substantial contributions to analysis or interpretation of data: $L P, R R, Y E, M M, P G S$; Final approval of the version published: $L P, R R, Y E, M M, U Z, C M R$, EMO, KAE, DLB, PGS.

Funding The REACH Registry is endorsed by the World Heart Federation. The REACH Registry was supported by Sanofi-Aventis, Bristol-Myers Squibb, and the Waksman Foundation (Tokyo, Japan).

Competing interests Dr LP reports grants and personal fees from Sanofi, personal fees from Servier, outside the submitted work; Dr RR reports grants and personal fees from Sanofi, personal fees from AstraZeneca, personal fees and non-financial support from Novartis, personal fees from MSD, outside the submitted work; M. YE has nothing to disclose; Dr MM reports grants and personal fees from MSD, Novartis, Novo Nordisk, Sanofi and Servier, personal fees and non-financial support from Abbott, Intarcia, and Eli Lilly, outside the submitted work; Dr UZ reports personal fees from Astra Zeneca, personal fees from Boehringer Ingelheim, personal fees from Novartis, personal fees from BMS, personal fees from Sanofi, personal fees from MSD, personal fees from Pfizer, outside the submitted work; Dr. Reid reports grants from Sanofi Aventis, during the conduct of the study; Dr MO reports personal fees from Abbott Vascular, personal fees from Abiomed, personal fees from Astra Zeneca, 
personal fees from Biotie, personal fees from Boehringer Inelheim, personal fees from Bristol Myers Squibb, grants and personal fees from Daiichi Sankyo, personal fees from Faculty Connection, grants from Gilead Sciences, grants and personal fees from Janssen Parmaceuticals, personal fees from Merck, personal fees from Sanofi Aventis, personal fees from St. Jude Medical, personal fees from Stealth eptides, personal fees from The Medicines Company, personal fees from Medscape, outside the submitted work; Dr KAE has nothing to disclose; D.L.B. reports grants from Bristol Myers Squibb, grants from Sanofi Aventis, during the conduct of the study; grants from Amarin, grants from AstraZeneca, grants from Bristol-Myers Squibb, grants from Eisai, grants from Ethicon, grants from Medtronic, grants from sanofi aventis, grants from The Medicines Company, other from FlowCo, other from PLx Pharma, other from Takeda, personal fees from Duke Clinical Research Institute, Personal fees from Mayo Clinic, personal fees from Population Health Research Institute, personal fees and non-financial support from American College of Cardiology, personal fees from Belvoir Publications, personal fees from Slack Publications, personal fees from WebMD, personal fees from Elsevier, other from Medscape Cardiology, other from Regado Biosciences, other from Boston VA Research Institute, personal fees and non-financial support from Society of Cardiovascular Patient Care, non-financial support from American Heart Association, personal fees from HMP Communications, grants from Roche, personal fees from Harvard Clinical Research Institute, other from Clinical Cardiology, personal fees from Journal of the American College of Cardiology, other from VA, grants from Pfizer, grants from Forest Laboratories, grants from Ischemix, other from St. Jude Medical, other from Biotronik, other from Cardax, other from Ameri can College of Cardiology, other from Boston Scientific, grants from Amgen, outside the submitted work; Dr PG reports personal fees from BMS and Sanofi, during the conduct of the study; grants from Merck, Sanofi, and Servier, personal fees from Amarin, AstraZeneca, Bayer, Boehringer-Ingelheim, Bristol-MyersSquibb, CSL-Behring, Daiichi-Sankyo, GlaxoSmithKline, Janssen, Lilly, Merck Novartis, Pfizer, Regeneron, Sanofi, Servier, The M edicines Company, outside the submitted work.

Provenance and peer review Not commissioned; externally peer reviewed.

(c) Article author(s) (or their employer(s) unless otherwise stated in the text of the article) 2017. All rights reserved. No commercial use is permitted unless otherwise expressly granted.

\section{REFERENCES}

1 Lévy BI. Can angiotensin II type 2 receptors have deleterious effects in cardiovascular disease? implications for therapeutic blockade of the renin-angiotensin system. Circulation 2004;109:8-13.

2 Bangalore S, Fakheri R, Toklu B, et al. Angiotensin-Converting enzyme inhibitors or angiotensin receptor blockers in patients without heart failure? insights from 254,301 patients from randomized trials. Mayo Clin Proc 2016;91:51-60.

3 Fihn SD, Gardin JM, Abrams J, et al; American College of Cardiology Foundation/ American Heart Association Task Force. 2012 ACCF/AHA/ACP/AATS/PCNA/SCAI/ STS guideline for the diagnosis and management of patients with stable ischemic heart disease: a report of the American college of cardiology foundation/American heart association task force on practice guidelines, and the american college of physicians, american association for thoracic surgery, preventive cardiovascular nurses association, society for cardiovascular angiography and interventions, and society of thoracic surgeons. Circulation 2012;126:e354-e471.

4 Montalescot G, Sechtem U, Achenbach S, et al; Task Force MembersESC Committee for Practice GuidelinesDocument Reviewers. ESC guidelines on the management of stable coronary artery disease: the task force on the management of stable coronary artery disease of the european society of cardiology. Eur Heart J 2013;34:2949-3003.

5 Dickstein K, Kjekshus J; OPTIMAAL Steering Committee of the OPTIMAAL Study Group. Effects of losartan and captopril on mortality and morbidity in high-risk patients after acute myocardial infarction: the OPTIMAAL randomised trial. optimal trial in myocardial infarction with angiotensin II antagonist losartan. Lancet 2002;360:752-60.

6 Pfeffer MA, McMurray JJ, Velazquez EJ, et al; Valsartan in Acute Myocardial Infarction Trial Investigators. Valsartan, captopril, or both in myocardial infarction complicated by heart failure, left ventricular dysfunction, or both. N Engl J Med 2003;349:1893-906.

7 Barnett AH, Bain SC, Bouter P, et al; Diabetics Exposed to Telmisartan and Enalapril Study Group. Angiotensin-receptor blockade versus converting-enzyme inhibition in type 2 diabetes and nephropathy. N Engl J Med 2004;351:1952-61.

8 Yusuf S, Teo KK, Pogue J, et al; ONTARGET Investigators. Telmisartan, ramipril, or both in patients at high risk for vascular events. N Engl J Med 2008;358:1547-59.
9 Montalescot G, Drexler H, Gallo R, et al. Effect of irbesartan and enalapril in nonST elevation acute coronary syndrome: results of the randomized, double-blind ARCHIPELAGO study. Eur Heart J 2009;30:2733-41.

10 Bhatt DL. Advancing the care of cardiac patients using registry data: going where randomized clinical trials dare not. Jama 2010;303:2188-9.

11 Benson K, Hartz AJ. A comparison of observational studies and randomized, controlled trials. N Engl J Med 2000;342:1878-86.

12 Rothwell PM. External validity of randomised controlled trials: "to whom do the results of this trial apply?". Lancet 2005;365:82-93.

13 Bangalore S, Steg G, Deedwania P, et al; REACH Registry Investigators. $\beta$-Blocker use and clinical outcomes in stable outpatients with and without coronary artery disease. Jama 2012;308:1340-9.

14 Sorbets E, Labreuche J, Simon T, et al. Renin-angiotensin system antagonists and clinical outcomes in stable coronary artery disease without heart failure. Eur Heart $J$ 2014;35:1760-8.

15 van Vark LC, Bertrand M, Akkerhuis KM, et al. Angiotensin-converting enzyme inhibitors reduce mortality in hypertension: a meta-analysis of randomized clinical trials of renin-angiotensin-aldosterone system inhibitors involving 158,998 patients. Eur Heart J 2012;33:2088-97.

16 Cheng J, Zhang W, Zhang $X$, et al. Effect of angiotensin-converting enzyme inhibitors and angiotensin II receptor blockers on all-cause mortality, cardiovascular deaths, and cardiovascular events in patients with diabetes mellitus: a meta-analysis. JAMA Intern Med 2014;174:773-85.

17 Emdin CA, Rahimi K, Neal B, et al. Blood pressure lowering in type 2 diabetes: a systematic review and meta-analysis. Jama 2015;313:603-15.

18 Palmer SC, Mavridis D, Navarese E, et al. Comparative efficacy and safety of blood pressure-lowering agents in adults with diabetes and kidney disease: a network meta-analysis. Lancet 2015;385:2047-56.

19 Bhatt DL, Steg PG, Ohman EM, et al; REACH Registry Investigators. International prevalence, recognition, and treatment of cardiovascular risk factors in outpatients with atherothrombosis. Jama 2006:295:180-9.

20 Parsons L, 2004. Performing a 1:n case-control match on propensity score. TwentyNinth Annual SAS® Users Group International (SUGI) Conference Invited Papers 165-29.

21 Yusuf S, Sleight P, Pogue J, et al. Effects of an angiotensin-converting-enzyme inhibitor, ramipril, on cardiovascular events in high-risk patients. the heart outcomes prevention evaluation study investigators. N Engl J Med 2000;342:145-53.

22 Dahlöf B, Devereux RB, Kjeldsen SE, et al; LIFE Study Group. Cardiovascular morbidity and mortality in the losartan intervention for endpoint reduction in hypertension study (LIFE): a randomised trial against atenolol. Lancet 2002;359:995-1003.

23 Fox KM; EURopean trial On reduction of cardiac events with Perindopril in stable coronary Artery disease Investigators. Efficacy of perindopril in reduction of cardiovascular events among patients with stable coronary artery disease: randomised, double-blind, placebo-controlled, multicentre trial (the EUROPA study). Lancet 2003;362:782-8.

24 Yusuf S, Diener HC, Sacco RL, et al; PRoFESS Study Group. Telmisartan to prevent recurrent stroke and cardiovascular events. N Engl J Med 2008;359:1225-37.

25 Pitt B, Segal R, Martinez FA, et al. Randomised trial of losartan versus captopril in patients over 65 with heart failure (evaluation of losartan in the elderly study, ELITE). Lancet 1997;349:747-52.

26 Pitt B, Poole-Wilson PA, Segal R, et al. Effect of losartan compared with captopril on mortality in patients with symptomatic heart failure: randomised trial--the losartan heart failure survival study ELITE II. Lancet 2000;355:1582-7.

27 Ponikowski P, Voors AA, Anker SD, et al; Authors/Task Force Members. 2016 ESC guidelines for the diagnosis and treatment of acute and chronic heart failure: the task force for the diagnosis and treatment of acute and chronic heart failure of the european society of cardiology (ESC)Developed with the special contribution of the heart failure association (HFA) of the ESC. Eur Heart J 2016;37:2129-200.

28 Packer M, Poole-Wilson PA, Armstrong PW, et al. Comparative effects of low and high doses of the angiotensin-converting enzyme inhibitor, lisinopril, on morbidity and mortality in chronic heart failure. ATLAS study group. Circulation 1999;100:2312-8.

29 Marre M, Lievre M, Chatellier G, et al; DIABHYCAR Study Investigators. Effects of low dose ramipril on cardiovascular and renal outcomes in patients with type 2 diabetes and raised excretion of urinary albumin: randomised, double blind, placebo controlled trial (the DIABHYCAR study). Bmj 2004;328:495.

30 Konstam MA, Neaton JD, Dickstein K, et al; HEAAL Investigators. Effects of high-dose versus low-dose losartan on clinical outcomes in patients with heart failure (HEAAL study): a randomised, double-blind trial. Lancet 2009;374:1840-8. 